\title{
Effects of memory reactivation treatments on postdiscrimination generalization performance in pigeons
}

\author{
THOMAS B. MOYE and DAVID R. THOMAS \\ University of Colorado at Boulder, Boulder, Colorado
}

\begin{abstract}
In Experiment 1, it was shown that generalization testing following successive discrimination training between two closely spaced wavelengths results in a sharp gradient with a peak of responding shifted from S+ so as to be further removed from S-. Testing after a 24-h delay resulted in a flatter gradient with greater peak (and area) shift. A 5-min pretest exposure to S+, reinforced or unreinforced, or to $\mathbf{S}$ - (unreinforced) reinstated immediate test performance; free reinforcement with no discriminative stimulus present had no such effect. Experiment 2 replicated the flattening of generalization gradients and enhanced peak shift in delayed testing. Free feeding in a pretest treatment with a distinctive food uniquely associated with the wavelength discrimination problem failed to reinstate immediate test performance. Experiment 3 tested the hypothesis that free feeding failed as a reactivation treatment because it did not engender keypecking. Subjects were trained to peck a vertical line stimulus before being given wavelength discrimination training. Again, the enhanced peak shift and greater flattening with delayed wavelength generalization testing was found. A pretest exposure to the vertical line stimulus elicited pecking but had no effect on subsequent wavelength generalization. Thus, only a reactivation treatment that included one of the discrimination training stimuli was effective in producing delayed test performance comparable to that obtained in an immediate test.
\end{abstract}

The analysis of factors that alleviate forgetting has been an area of prime importance for investigators of animal memory (e.g., Campbell \& Jaynes, 1966; Lewis, 1979; Spear, 1973, 1976; Spear \& Parsons, 1976). The general strategy has been to train subjects on some task, expose them to a source of forgetting (such as the passage of time or intervening events), and then test for retention of the original task after some memory reactivation manipulation. When reactivation treatments improve retention performance relative to that of subjects not provided with such treatments, forgetting is said to have been alleviated (cf. Spear, 1976).

Reactivation treatments usually consist of the presentation of some isolated aspect of the original training situation shortly before retention testing. Exposure to aspects of earlier training is thought to facilitate the retrieval of the memory of that training, thus improving performance on the subsequent retention test.

The present experiments examined the effects of various pretest treatments on delayed postdiscrimina-

Requests for reprints should be addressed to David R. Thomas, Department of Psychology, Campus Box 345, University of Colorado at Boulder, Boulder, Colorado 80309. This research was supported by NSF Research Grant BNS-7801407. It was reported at the April 1981 meeting of the Rocky Mountain Psychological Association in Denver. tion generalization test performance in pigeons. Several studies have demonstrated that delays between training and testing have systematic effects on generalization test performance. Perkins and Weyant (1958) trained rats to run an alley to obtain food, and subsequently measured generalization by varying the color of the alley used. Testing was carried out either immediately or 1 week after training. The generalization gradients obtained 1 week following training were reliably flatter than those obtained immediately. Similarly, Thomas and Lopez (1962) found that pigeons tested for wavelength generalization either $24 \mathrm{~h}$ or 1 week following single stimulus keypeck training exhibited flatter generalization gradients than did subjects tested immediately after training. Thomas (1981) has suggested that the flattening of generalization gradients with the passage of time reflects forgetting of the stimulus value used in training.

Thomas and Burr (1969) obtained generalization gradients at different times following the completion of intradimensional discrimination training. Three groups of pigeons were trained to discriminate between a 550-nm keylight S+ (VI reinforced) and a 570-nm keylight S - (extinguished). Subjects in one group were tested for wavelength generalization immediately following attainment of the discrimination criterion, while subjects in the other two groups 
were tested after a 24-h delay. Subjects in one of the delay groups were given a 3-min reinforced warmup with the $\mathrm{S}+$ from earlier training prior to testing, while subjects in the other delay group were tested with no warm-up. The delay group with no warm-up revealed forgetting according to two different measures. Their gradient was reliably flatter and displayed greater peak shift than that of the group tested immediately. By contrast, the gradient of the group given a brief warm-up was virtually indistinguishable from that obtained in the immediate test. This result demonstrates that pretest experience with a subset of the cues from earlier discrimination training ( $\mathrm{S}+$ and reinforcement) is sufficient to alleviate the effect of the 24-h delay between training and testing. The pretest warm-up may be viewed as a reminder treatment, serving to reactivate the memory of the earlier training conditions.

Although Thomas and Burr (1969) demonstrated the effectiveness of a pre-generalization test warm-up in eliminating the effects of a delay between training and testing, they did not isolate which aspect(s) of the warm-up procedure were responsible for the improved retention performance. The present experiments examined the effects of pretest treatments consisting of the presentation of more restricted aspects of earlier training in an attempt to isolate the cues responsible for the elimination of the effects of a 24-h delay between training and generalization testing.

\section{EXPERIMENT 1}

This experiment examined the effects of several different pretest treatments administered immediately prior to delayed generalization testing. The effectiveness of a particular treatment was judged against the performance of nontreated control groups tested immediately or $24 \mathrm{~h}$ after training. The pretest treatments employed in this experiment consisted of: (1) experience with the $S+$ from discrimination training reinforced on a variable interval schedule; (2) experience with the $\mathrm{S}+$ without reinforcement available; (3) experience with the $S$ - from discrimination training, and (4) free reinforcement delivered in the absence of any discriminative stimuli. Treatments which are effective in eliminating the effects of the delay should result in generalization test performance similar to that found in subjects tested immediately. Ineffective treatments should result in test performance more like that found in control subjects tested following a delay.

\section{Method}

Subjects. The subjects were 48 naive adult pigeons maintained at approximately $75 \%$ of their free-feeding weights during the experiment. The birds were individually housed, with grit and water continuously available. They were maintained on a 16-h-light/ 8-h-dark cycle.

Apparatus. Four standard sound-attenuating pigeon chambers with interior dimensions of $33.7 \times 34.3 \times 29.8 \mathrm{~cm}(\mathrm{~L} \times \mathrm{W} \times \mathrm{H})$ were used. Each chamber had a wire-mesh floor, and one wall, constructed of aluminum, had a 2.5 -cm-diam translucent response key centered $20.3 \mathrm{~cm}$ above the floor. The hopper aperture, located $7.3 \mathrm{~cm}$ above the floor, was $5.7 \times 4.4 \mathrm{~cm}(\mathrm{~W} \times \mathrm{H})$. The aperture allowed access to grain from a solenoid-operated hopper. An ESB 24-V lamp located above the hopper was illuminated during grain presentation.

Stimuli were projected onto a translucent screen on the front of IEE in-line display cells located $.63 \mathrm{~cm}$ behind the response key. The display cells, fitted with Kodak-Wratten gelatin filters Nos. $75,74,99,73$, and $72 b$, were combined to provide chromatic stimuli with nominal peak wavelengths of $490,538,547,555$, 560, 576, 591, and $606 \mathrm{~nm}$ (cf. Lyons \& Klipec, 1971). The display cells also allowed projection of a $.16 \mathrm{~cm}$ wide $\times 2.54 \mathrm{~cm}$ long white line on a black background with a 90 -deg (vertical) orientation. Projection of these stimuli was accomplished by No. 1815 (14-V) miniature lamps.

Masking noise was delivered through a $10.2-\mathrm{cm}$-diam speaker mounted to the right of the hopper. A ventilation fan provided additional masking noise. The noise level in the chamber was measured at $70 \mathrm{~dB}(\mathrm{SPL})$.

Procedure. On Day 1, the subjects in all groups were hoppertrained and hand-shaped to peck the key on which a $560-\mathrm{nm}$ stimulus was presented. The subjects were then allowed to earn 60 reinforcers (each reinforcer consisted of 3 -sec access to grain) on a schedule gradually increased from continuous reinforcement to a constant probability (VI) 30-sec schedule (Catania \& Reynolds, 1968). On Day 2, the subjects were allowed to earn 50 reinforcers for pecking at the $560-\mathrm{nm}$ stimulus on the same gradually increasing schedule used on Day 1. On Day 3, discrimination training was begun for all subjects. Responding in the presence of the 560-nm stimulus $(S+$ ) was reinforced on a constant probability (VI) 30-sec schedule, while responding in the presence of the 576-nm stimulus (S-) was extinguished. The stimulus periods were $55 \mathrm{sec}$ in duration and separated by 5 -sec blackout periods. Fifteen $\mathrm{S}+$ and $\mathrm{S}$ - periods were presented in each session in pseudorandom order, with the constraint that no more than three $\mathrm{S}+$ or three $\mathrm{S}-$ periods occur consecutively. The subjects were given one session per day for at least 3 days and until a performance criterion ( $90 \%$ of total responses emitted during a session in the presence of $\mathrm{S}+$ ) was achieved.

Following attainment of the performance criterion, each subject was tested for wavelength generalization in extinction. Eight wavelengths $(490,538,547,555,560,576,591$, and $606 \mathrm{~nm})$ were presented once in each of eight randomized blocks. The stimulus periods were $30 \mathrm{sec}$ in duration, and were separated by 5 -sec blackouts. Subjects in Group Immediate Test were tested 1 min following the end of the session in which they had achieved the performance criterion. During the minute between training and testing, these subjects remained in the chambers with no key stimulus present. Subjects in all the other groups were tested $24 \mathrm{~h}$ following attainment of criterion performance. The subjects in Groups S+ RF-REM, S+ No RF-REM, and S- REM were presented with a 5 -min treatment period immediately prior to generalization testing. Group S+ RF-REM subjects were presented with the $560-\mathrm{nm} \mathrm{S}+$ stimulus, and were reinforced for keypecking on a VI 30-sec schedule. Subjects in Group S+ No RF-REM were presented with the $560-\mathrm{nm} S+$, but responses were not reinforced. Subjects in Group $S-$ REM were presented with the 576-nm S-, and keypecking was not reinforced. Stimulus-on periods during these pretest treatment conditions were $55 \mathrm{sec}$ in duration and were separated by 5 -sec blackout periods. Group VT-REM subjects were placed in the chambers for $5 \mathrm{~min}$ with no key stimulus present, but were presented with free reinforcement on a variable-time 30 -sec schedule (several subjects in this condition were observed during this treatment, 
and they readily ate from the hopper). Subjects in Group No Warm-up were merely placed in the chamber (with the white noise on) for $5 \mathrm{~min}$ with no key stimulus or reinforcement present. The pretest treatments and testing were separated by a 5 -sec blackout.

\section{Results and Discussion}

The mean number of days required to achieve the discrimination criterion were $6.0,5.6,6.3,5.1,5.8$, and 5.8 for Groups Immediate Test, S+ RF-REM, S+ No RF-REM, S- REM, VT-REM, and No Warm-up, respectively. These means did not differ significantly $[F(5,42)<1.0]$. The mean number of responses emitted during generalization testing likewise did not differ significantly among the groups, with a range of 540.8 responses, for Group Immediate Test, to $1,026.5$ responses, for VT-REM $[F(5,42)=1.20]$.

The relative wavelength generalization gradients based on the percentage of total responses to each test stimulus value are presented in Panels $a$ and $\mathrm{b}$ of Figure 1. As is typical following intradimensional discrimination training between closely spaced values (see Purtle, 1973), the point of maximal responding for each gradient is shifted away from S+ $(560 \mathrm{~nm})$ to a value farther removed from $\mathrm{S}-$ $(576 \mathrm{~nm})$. In the left panel (Panel a) are the generalization gradients obtained from Groups Immediate
Test, S+ RF-REM, and No Warm-up. These three groups constitute a replication of the Thomas and Burr (1969) experiment. The gradients for the Immediate Test and S+ RF-REM groups both peak at the $555-\mathrm{nm}$ value, while the gradient of the No Warm-up group peaks at the $547-\mathrm{nm}$ value, showing greater peak shift. As in the Thomas and Burr (1969) experiment, a brief treatment administered prior to delayed generalization testing consisting of reinforced experience with the $\mathrm{S}+$ from discrimination training, is capable of restoring test performance to a level comparable to that obtained immediately after training (as measured by peak shift).

In Panel $b$, the gradients of the other treatment groups are presented along with the gradient of Group S+ RF-REM, included for comparison purposes. Groups S+ No RF-REM and S-REM both produced gradients similar to that of Group $S+R F$. REM, with peaks at the 555-nm stimulus value. Group VT-REM produced a gradient similar to that of the No Warm-up group, with maximal responding occurring to the $547-\mathrm{nm}$ stimulus.

These impressions were confirmed statistically. In order to compare the location of the generalization gradients, the amount of peak shift was converted to amount of area shift, a continuous measure. Each individual subject's gradient was treated as a grouped

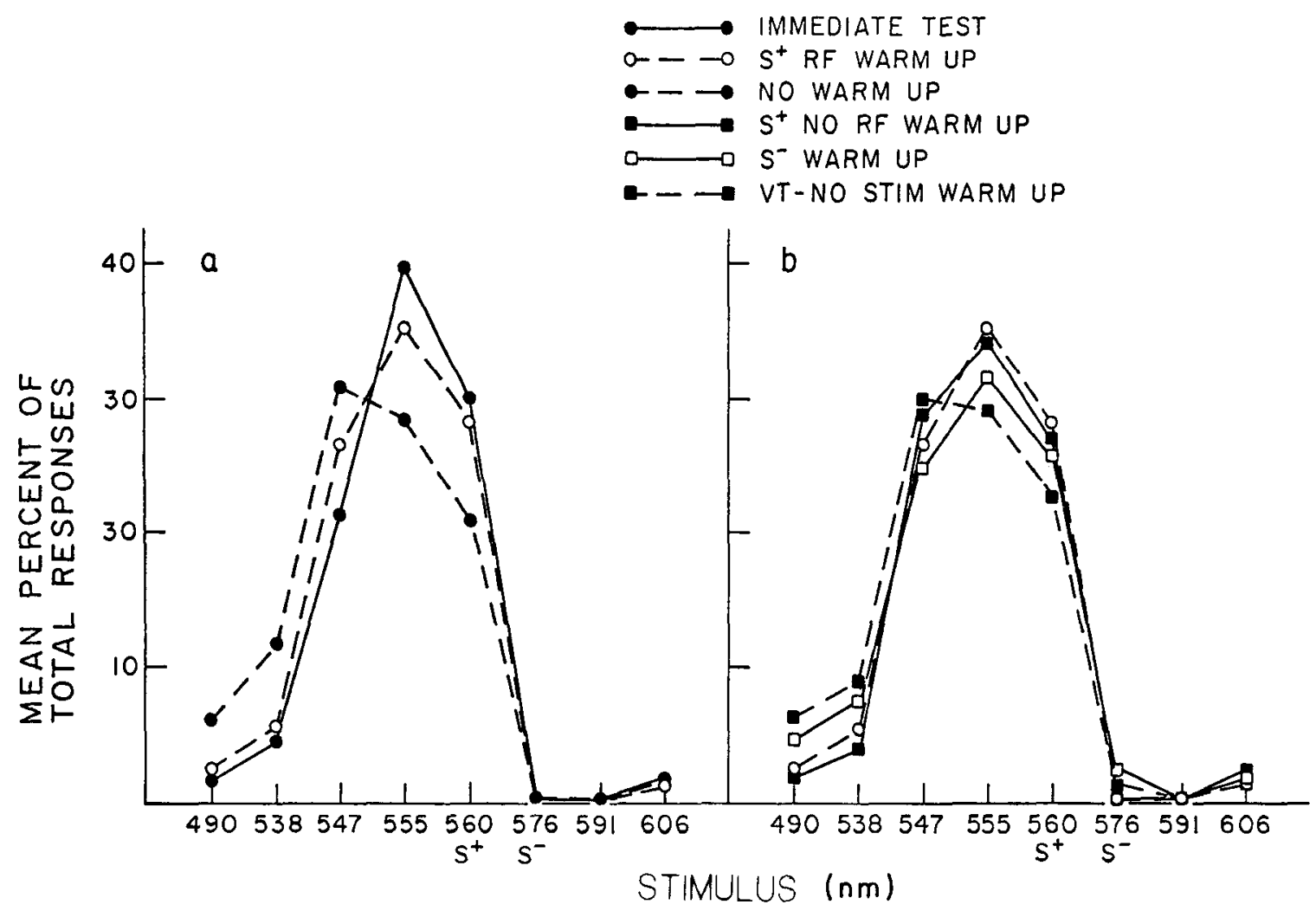

Figure 1. Mean relative stimulus generalization gradients obtained either immediately or after a delay subsequent to wavelength discrimination training in Experiment 1. 
frequency distribution. The generalization test values of 606 through $490 \mathrm{~nm}$ were each assigned an integer from 1 to 8 , respectively. Each integer was multiplied by the number of responses made to the corresponding stimulus value during generalization testing for each subject; then the mean of the resulting distribution was computed. Thus, a larger value indicated a greater amount of area shift in the gradient. The gradient means for each group computed in this manner were $4.95,5.05,5.12,5.13,5.34$, and 5.45 for Groups Immediate Test, S+ No RF-REM, S- REM, S + RF-REM, VT-REM, and No Warm-up, respectively. Analysis of variance revealed a significant effect of group $[F(5,42)=6.22, p<.01]$. Subsequent Newman-Keuls post hoc comparisons $(\alpha=.05)$ indicated that Groups Immediate Test, S+ RF-REM, S+ No RF-REM, and S-REM did not differ among themselves, but did differ from Groups VTREM and No Warm-up, which did not differ from each other. Thus, the pretest treatments consisting of reinforced experience with the $S+$ from discrimination training, nonreinforced experience with the $S+$, or experience with the $S-$ were each sufficient to restore generalization performance in the delayed test subjects to a level comparable to that found in subjects tested only 1 min following discrimination training, as measured by peak shift or area shift.

The slopes of the generalization gradients were also compared. Since different subjects produced gradients with peaks at different stimulus values, the percent of total responses at the peak of each individual subject's gradient was used as the measure of slope. Group means computed from the individual peaks were $40.2 \%, 37.6 \%, 35.5 \%, 33.8 \%, 33.0 \%$, and $32.5 \%$ of total responses for Groups Immediate Test, S+ No RF-REM, S+ RF-REM, S- REM, No Warm-up, and VT-REM, respectively. Analysis of variance revealed that there was a significant effect of group $[\mathrm{F}(4,42)=2.53, \mathrm{p}<.05]$. However, NewmanKeuls comparisons $(\alpha=.05)$ indicated that the only difference between groups that approached statistical significance was that between Groups Immediate Test and VT-REM, the extreme values. Although none of the individual post hoc comparisons reached significance, the differences in slope were consistent with earlier results (i.e., Thomas and Burr, 1969). Groups Immediate Test and S+ RF-REM produced gradients sharper than those of the No Warm-up group, which is what was found by the Thomas and Burr (1969) study. The gradients of Groups S+ No RF-REM and S- REM were sharper than those of either the VT-REM or No Warm-up groups, which is consistent with the results obtained with the area shift measure.

In summary, reexposure to one of the stimulus values from earlier intradimensional discrimination training was sufficient to restore delayed generalization performance to a level equivalent to that ob- tained in subjects tested only $1 \mathrm{~min}$ following training. On the other hand, pretest exposure to the reinforcer appears to have had no effect on delayed generalization test performance. It is interesting that nonreinforced experience with the $\mathrm{S}+$ from discrimination training was an effective treatment. This condition was inconsistent with the training situation, since responding to the $\mathrm{S}+$ was reinforced on a fairly dense schedule (VI-30 sec) during training, but was extinguished during the pretest treatment. It is possible that the duration of the treatment was sufficient to reactivate the memory of the conditions from earlier training, but insufficient to produce new learning about the stimulus. A longer pretest treatment might have produced interference rather than facilitation. Work by Gordon, Smith, and Katz (1979) has demonstrated such a dual effect of extinction trials on the retention of active avoidance training in rats. A short period of extinction prior to retention testing enhanced performance, while a longer period of extinction interfered with later performance.

\section{EXPERIMENT 2}

In Experiment 1, the pretest presentation of free reinforcement without any discriminative stimulus present was an ineffective treatment. This result may be contrasted with those of other studies which have shown that noncontingent preretention test exposure to the aversive reinforcer (e.g., Hamberg \& Spear, 1978; Klein \& Spear, 1970; Miller \& Springer, 1972) or to the appetitive reinforcer used in training (Miller, Ott, Berk, \& Springer, 1974) is an effective memory reactivation treatment. In Experiment 1, the reinforcer would not have been uniquely associated with the target discrimination, because subjects were given supplemental feedings in the home cages consisting of the same food (mixed grain) used in the experimental chambers. Studies that have shown the effectiveness of reinforcement as a reactivation treatment generally did not allow access to the particular reinforcer employed in the home cages.

The purpose of Experiment 2 was to investigate whether the use of a food reinforcer distinct from that used in supplemental feedings would allow it to serve as an effective pretest reminder.

\footnotetext{
Method

Subjects. The subjects were 24 experimentally naive adult pigeons maintained as in Experiment 1.

Apparatus. The apparatus was the same as that used in Experiment 1 .

Procedure. The subjects were hopper-trained and hand-shaped to peck the 560-nm stimulus, as in Experiment 1. Discrimination training was carried out as in Experiment 1, with the exception that the food reinforcer was changed from mixed grain to Purina Pigeon Chow Checkers beginning on the first day of discrimination training. ${ }^{1}$ Following attainment of the discrimination criterion $(90 \%$ of total responses emitted during a session in the
} 
presence of $\mathbf{S}+$ ), each subject was tested for wavelength generalization in extinction, as in Experiment 1. The subjects in Group Immediate Test were tested 1 min following the end of the session in which they achieved the performance criterion. The subjects in the other two groups were tested $24 \mathrm{~h}$ following attainment of the criterion. Immediately prior to testing, the subjects in Group VT-REM were placed in the experimental chambers and presented with free reinforcement (still consisting of the Pigeon Chow Checkers) on a variable-time $30-\mathrm{sec}$ schedule for $5 \mathrm{~min}$. Subjects in Group No Warm-up were merely placed in the chambers for $5 \mathrm{~min}$ with no key stimulus or reinforcement present.

\section{Results and Discussion}

The mean number of days required to master the discrimination problem were $4.9,5.1$, and 5.3 for Groups Immediate Test, No Warm-up, and VTREM, respectively. The differences between these means were not significant $[\mathrm{F}(2,21)<1.0]$. The number of responses emitted during generalization testing averaged 546.9, 713.8, and 801.1 for Groups Immediate Test, VT-REM, and No Warm-up. Again, the differences between these means did not reach statistical significance $[\mathrm{F}(2,21)=2.09, \mathrm{p}=.15]$.

The relative wavelength generalization gradients obtained in Experiment 2 are presented in Figure 2. As in Experiment 1, the gradients of all groups displayed peak shift. The gradient of Group Immediate Test peaked sharply at the 555-nm stimulus value. The gradients produced by Groups VT-REM and No Warm-up exhibit nearly equal responding to the 555- and 547-nm values. Statistical analysis was again performed on the area shift measure, computed as in Experiment 1. The means were 5.02, 5.47, and

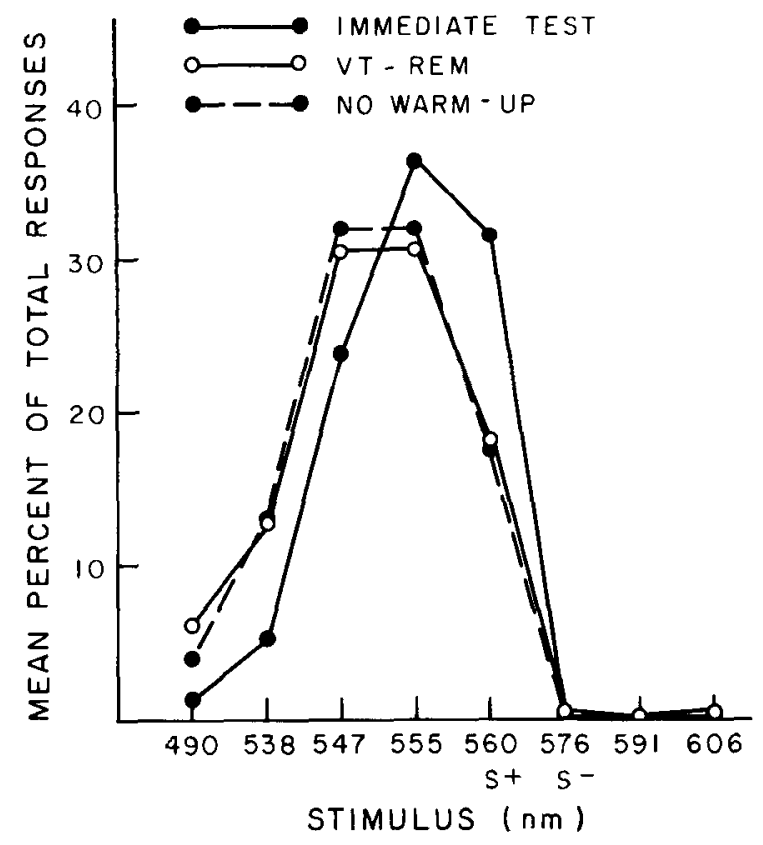

Figure 2. Mean relative stimulus generalization gradients obtained either immediately or after a delay subsequent to wavelength discrimination training employing a distinctive reinforcer in Experiment 2.
5.50 for Groups Immediate Test, No Warm-up, and VT-REM, respectively. Analysis of variance indicated a significant effect of group membership $[F(2,21)=16.88, p<.001]$. Subsequent NewmanKeuls comparisons $(\alpha=.05)$ showed that the gradient of Group Immediate Test showed reliably less of a shift than did those of either Group No Warm-up or VT-REM, which did not differ from one another.

The slopes of the gradients were also compared, as in Experiment 1, with the percent of total responses at the peak of each individual subject's gradient used as the measure of gradient slope. The means were $38.2 \%, 33.5 \%$, and $33.1 \%$ of total responses for Groups Immediate Test, No Warm-up, and VTREM, respectively. Analysis of variance revealed a significant effect of group $[F(2,21)=6.80, p<.005]$. Newman-Keuls comparisons $(\alpha=.05)$ indicated that the gradient of Group Immediate Test was sharper than those of either the VT-REM group or the No Warm-up group, which did not differ from each other. We may conclude that reinforcer presentation prior to delayed generalization testing is not sufficient to restore performance to a level comparable to that obtained immediately following training. This is true if the reinforcer is the same food fed to the subjects in the home cages (Experiment 1) or if a distinctively different type of food is employed in the experimental chambers (Experiment 2).

\section{EXPERIMENT 3}

A common feature of the effective pretest treatments in Experiment 1 was the fact that some responding occurred to an on-key stimulus prior to testing. Even in the group presented with the Sfrom earlier discrimination training, the subjects did emit some responses. No keypeck responses were made, however, under the free-food conditions of Experiments 1 and 2. Perhaps allowing subjects to respond (regardless of the stimulus present) in the experimental chambers prior to testing is sufficient to restore delayed performance to a level comparable to that obtained immediately after training. Experiment 3 was performed to examine this possibility.

\section{Method}

Subjects. The subjects vere 24 experimentally naive adult pigeons maintained as in Experiments 1 and 2.

Apparatus. The apparatus was the same as that used in Experiments 1 and 2.

Procedure. On Day 1, all subjects were hopper-trained and hand-shaped to peck a 90-deg (vertical) white line on a dark key. The subjects were then allowed to earn 60 reinforcers on a schedule gradually increased from continuous reinforcement to a constant probability (VI) 30-sec schedule. On Day 2, the subjects were allowed to earn 50 reinforcers for pecking at the 90 -deg line on the same gradually increasing schedule as on Day 1 . Beginning on the 3rd day, all subjects were given five daily sessions of training with the vertical line. Each session consisted of $\mathbf{3 0}$ stimulus periods of $55-\mathrm{sec}$ duration separated by 5 -sec blackouts. 
Reinforcement was available on a constant probability (VI) 30-sec schedule during half of the stimulus periods. Reinforced and unreinforced stimulus periods were arranged in pseudorandom order with the constraint that no more than three reinforced or unreinforced periods occur consecutively.

After the five sessions of line training, subjects in all groups received wavelength discrimination training identical to that in Experiment 1. Following attainment of the discrimination criterion ( $90 \%$ of total responses emitted during a session in the presence of $S+$ ), each subject was tested for wavelength generalization in extinction, as in Experiment 1 and 2. Subjects in Group Immediate Test were tested $1 \mathrm{~min}$ following the end of the session in which they achieved the performance criterion. Subjects in the other two groups were tested $24 \mathrm{~h}$ following attainment of the criterion. Group Line Warm-up was presented with the vertical line for five 55 -sec periods separated by 5 -sec blackouts immediately prior to wavelength testing. Reinforcement was unavailable during this treatment phase. The pretest treatment and the wavelength generalization test were separated by a 5-sec blackout. Subjects in Group No Warm-up were merely placed in the box for $5 \mathrm{~min}$ with no key stimulus or reinforcement present.

\section{Results and Discussion}

As in Experiments 1 and 2, there were no significant differences between groups in the number of sessions required to achieve the discrimination criterion; 5.0, 4.5, and 4.3 sessions were required for Groups Immediate Test, Line Warm-up, and No Warm-up, respectively $[\mathrm{F}(2,21)<1.0]$. The total number of responses emitted during generalization testing also did not differ between groups, with 792.1, 798.8, and 881.0 mean responses for Groups Immediate Test, Line Warm-up, and No Warm-up $[\mathrm{F}(2,21)<1.0]$.

The relative wavelength generalization gradients are presented in Figure 3. Again, all gradients show

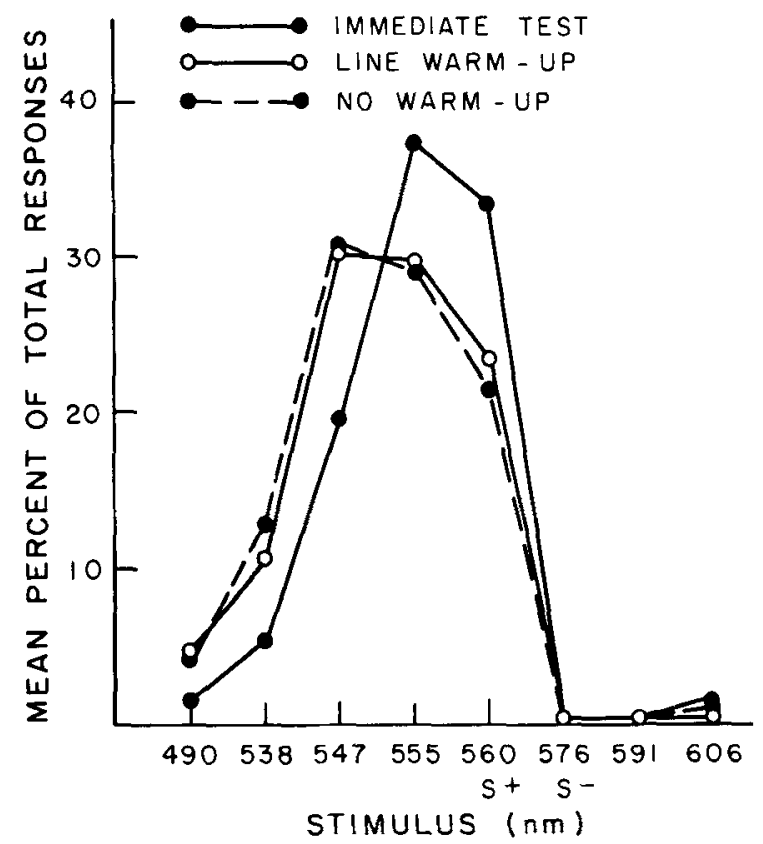

Figure 3. Mean relative stimulus generalization gradients obtained either immediately or after a delay subsequent to line training and wavelength discrimination training in Experiment 3 . the expected peak shift. The gradient of Group Immediate Test peaks at the $555-\mathrm{nm}$ stimulus value, while Groups Line Warm-up and No Warm-up both display peaks at the $547-\mathrm{nm}$ value. Area shift was computed for each of the gradients, as in Experiments 1 and 2. The resulting means were 4.94, 5.34, and $\mathbf{5 . 4 0}$ for Groups Immediate Test, Line Warm-up, and No Warm-up, respectively. Analysis of variance indicated a reliable effect of group $[F(2,21)=9.27$, $\mathrm{p}<.01]$. Subsequent Newman-Keuls individual comparisons $(\alpha=.05)$ revealed that the gradient of Group Immediate Test displayed less area shift than the gradients of Groups Line Warm-up or No Warm-up. Groups Line Warm-up and No Warm-up did not differ from each other.

The slopes of the gradients were compared, as in Experiments 1 and 2. The mean percent of total responses to the peak were $38.4,34.3$, and 32.1 for Groups Immediate Test, Line Warm-up, and No Warm-up, respectively. Analysis of variance indicated only a marginally significant effect of group $[F(2,21)=3.14, .05<p<.10]$. Newman-Keuls comparisons $(\alpha=.05)$ detected no significant differences between any of the groups, although, again, the gradient of the Immediate Test group was the sharpest.

Subjects that responded to a line stimulus for 5 min prior to wavelength testing produced a generalization gradient nearly identical to that of subjects given no pretest treatment. Thus, allowing subjects to respond to a stimulus on a dimension orthogonal to wavelength was ineffective in restoring delayed wavelength generalization test performance to a level comparable to that obtained immediately after training.

\section{GENERAL DISCUSSION}

The purpose of the present experiments was to examine the effects of various pretest treatments on delayed postdiscrimination generalization performance in pigeons. In Experiment 1, control subjects tested $24 \mathrm{~h}$ following training produced (insignificantly) flatter gradients with greater area shift than did subjects tested immediately after training. However, subjects given a brief pretest treatment consisting of reinforced experience with the $S+$ from earlier discrimination training produced gradients statistically indistinguishable from those of subjects tested immediately after training. These results are in agreement with those obtained with similar procedures by Thomas and Burr (1969). Other groups demonstrated that treatments consisting of pretest experience with either the $\mathrm{S}+$ or $\mathrm{S}-$ stimulus value (nonreinforced) were also sufficient to restore delayed test performance to a level equivalent to that of subjects tested immediately. Subjects given pretest access to free reinforcement in the apparatus with no discriminative stimulus present generated 
gradients indistinguishable from those of control subjects given a delayed test with no pretest treatment. In Experiment 2, it was shown that the effectiveness of free reinforcement as a pretest treatment was not enhanced when the food obtained in the experimental chambers was distinct from that received in the home cages. Finally, in Experiment 3, it was shown that simply responding in the experimental chambers was not an important factor in determining the effectiveness of the various pretest treatments. Pigeons allowed to respond to a vertical line for $5 \mathrm{~min}$ prior to the delayed wavelength generalization test performed comparably to control subjects given no treatment prior to the delayed test. Thus, it seems that experience with one of the stimulus values from earlier discrimination training is the important factor in eliminating the effects of a delay on generalization test performance.

These experiments attest to the usefulness of employing two measures of forgetting rather than just one. In all three experiments, the gradient of the immediate test group showed reliably less area shift than did the gradient of a no-treatment delayed-test group. Whereas the immediate test group also yielded a sharper gradient in all three experiments, only in Experiment 2 was the generalization slope difference statistically significant. The reason for the insensitivity of the generalization slope measure may have to do with the amount of error inherent in the use of percent of total responses to the modal stimulus as a measure of generalization slope. Where the peak of the gradient is shifted from the $\mathrm{S}+$ value following intradimensional discrimination training, the stimulus value to which maximal responding would theoretically have occurred may fall between two of the values used in testing. The resulting cropped gradient yields a percent at the peak value which substantially underestimates the slope of the underlying gradient. The likelihood of this error's occurring is independent of the treatment condition, but it occurs sufficiently often to substantially increase withingroup variance, making statistical significance difficult to attain.

It is appropriate to ask why an effective reactivation treatment leads to a sharpening of the postdiscrimination generalization gradient and a reduction in its peak and area shift. This question is equivalent, of course, to asking why forgetting is reflected in the increased shift and flattening. The traditional explanation of the peak shift and gradient sharpening following intradimensional discrimination training is Spence's (1937) theory, which postulates an interaction of an excitatory generalization gradient around $\mathrm{S}+$ and an inhibitory gradient around $S-$. The further flattening and increased peak shift with a delay might result from greater flattening of the inhibitory gradient than of the excitatory gradient during the delay interval. There is no way within the framework of Spence's theory, however, to explain the equivalent effects of reinforced or nonreinforced exposure to $S+$ and exposure to $S-$ in eliminating the effects of the delay. It should be pointed out, as it was by Thomas (1981) and Thomas and Burr (1969), that the lack of a satisfactory explanation of the effects of delayed testing on generalization test performance does not preclude the effective use of generalization measures in the study of forgetting and related phenomena.

The present results seem quite compatible with Lewis's (1979) and Spear's (1978) notions of memory reactivation. Basically, they have proposed that stimuli noticed during learning become part of the memory of the training episode. After a delay, the presentation of those stimuli noticed during learning is thought to reactivate the entire memory of earlier training. Perhaps during discrimination training, the two stimulus values and their associated reinforcement schedules are stored in memory as a unitary experience. Pretest reexposure to one of the stimulus values could then reactivate the representation of the other stimulus value as well, resulting in delayed generalization test performance that is comparable to immediate test performance.

Unfortunately, this analysis leaves unanswered the question of why only the pretest presentation of the on-key stimuli from discrimination training was an effective treatment. It seems likely that the nature of the to-be-remembered task may determine the effectiveness of reactivation cues. In the Miller et al. (1974) study, the learning task for the rat was simply to find and drink from a tube containing sucrose solution. The measure of performance was the latency to complete 10 licks, and the successful reactivation treatment was $60 \mathrm{sec}$ of exposure to a dish containing sucrose solution. In the present experiments, the subjects were required to remember to respond to one of two closely spaced wavelength values. As the difficulty of the training problem increases, it may be that effective memory reactivation requires pretest experience with cues increasingly specific to the target memory and/or to a greater number of such cues.

\section{REFERENCES}

Campbell, B. A., \& Jaynes, J. Reinstatement. Psychological Review, 1966, 73, 478-480.

Catania, A. C., \& Reynolds, G. S. A quantitative analysis of the responding maintained by interval schedules of reinforcement. Journal of the Experimental Analysis of Behavior, 1968, 11, 327-383.

Gordon, W. C., Smith, G. J., \& Katz, D. S. Dual effects of response blocking following avoidance learning. Behavior Research and Therapy, 1979, 17, 479-487.

Hamberg, J. M., \& Spear, N. E. Alleviation of forgetting of discrimination learning. Learning and Motivation, 1978, 9, 466-476.

KLEIN, S. B., \& SPEAR, N. E. Reactivation of avoidance-learning memory in the rat after intermediate retention intervals. Journal of Comparative and Physiological Psychology, 1970, 72, 498-504. 
LEwis, D. J. Psychobiology of active and inactive memory. Psychological Bulletin, 1979, 86, 1054-1083.

Lyons, J., \& KuIPEC, W. D. Color mixing with Kodak Wratten filters. Journal of the Experimental Analysis of Behavior, 1971, 15, 232.

Mille R, R. R., Ott, C. A., Berk, A. M., \& Springer, A. D. Appetitive memory restoration after electroconvulsive shock in the rat. Journal of Comparative and Physiological Psychology, $1974,87,717-723$.

Miller, R. R., \& SPRingen, A. D. Induced recovery of memory in rats following electroconvulsive shock. Physiology \& Behavior, $1972,8,645-651$.

Perkins, C. C., Jr., \& Weyant, R. G. The interval between training and test trials as determiner of the slope of generalization gradients. Journal of Comparative and Physiological Psychology, 1958, 51, 596-600.

Purtle, R. Peak shift: A review. Psychological Bulletin, 1973, 80, 408-421.

Spear, N. E. Retrieval of memory in animals. Psychological Review, 1973, 80, 163-194.

SPEAR, N. E. Retrieval of memory: A psychobiological approach. In W. K. Estes (Ed.), Handbook of learning and cognitive processes (Vol. 4): Attention and memory. Hillsdale, N.J: Erlbaum, 1976.

SPEAR, N. E. The processing of memories: Forgetting and retention. Hillsdale, N.J: Erlbaum, 1978.

Spear, N. E., \& Parsons, P. J. Analysis of a reactivation treatment: Ontogenetic determinants of alleviated forgetting. In D. L.
Medin, W. A. Roberts, \& R. T. Davis (Eds.), Processes of animal memory. Hillsdale, N.J: Erlbaum, 1976.

Spence, K. W. The differential response in animals to stimuli varying within a single dimension. Psychological Review, 1937, 44, 430-444.

Tномаs, D. R. Studies of long-term memory in the pigeon. In N. E. Spear \& R. R. Miller (Eds.), Information processing in animals: Memory mechanisms. Hillsdale, N.J: Erlbaum, 1981.

Thом As, D. R., \& BurR, D. E. S. Stimulus generalization as a function of the delay between training and testing procedures: A reevaluation. Journal of the Experimental Analysis of Behavior, 1969, 12, 105-109.

Thomas, D. R., \& Lopez, L. J. The effect of delayed testing on generalization slope. Journal of Comparative and Physiological Psychology, 1962, 44, 541-544.

\section{NOTE}

1. Several subjects were observed during the first few reinforcer presentations following the change in food type. Each subject observed failed to eat the Purina Pigeon Chow Checkers on the first few hopper presentations. This reluctance to eat the novel food would indicate that the subjects were able to discriminate the two food types.

(Manuscript received September 1, 1981; revision accepted for publication November 17, 1981.) 